\title{
The implementation of a structured specialized consultation for psoriasis management
}

\author{
Jo Lambert ${ }^{\mathrm{a}}$, Ana Karina Alves De Medeiros ${ }^{\mathrm{a}}$, Astrid Van Reempts ${ }^{\mathrm{a}}$, Kim Van Langenhove ${ }^{\mathrm{a}}$, Judith Rossey ${ }^{\mathrm{a}}$, \\ Elfie Deprez ${ }^{\mathrm{b}}$, Lucia Van Poucke ${ }^{\mathrm{b}}$ and Lynda Grine ${ }^{\mathrm{a}}$
}

${ }^{a}$ Ghent University, Ghent, Belgium; 'bepartment of Dermatology, Ghent University Hospital, Ghent, Belgium

\section{ABSTRACT}

Objectives: Psoriasis is a chronic skin disease requiring a multidimensional approach, given its varying appearance, presence of comorbidities and complex treatment regimens. Psoriasis care is however often performed fragmented and, in case of flares, reactive with little integrated information on and for the patient. Literature suggests a multileveled approach of psoriasis, but the effects of its implementation have not yet been validated. The aim of this study is to analyze the impact of a multileveled psoriasis consultation format, named PsoPlus, which has been implemented since 2012 in the Department of Dermatology at Ghent University Hospital in Belgium.

Methods: The patient population was divided into two groups: one following the regular consultation and one following the PsoPlus format. Demographic data, clinical outcome and treatment approach of psoriasis patients were compared.

Results: Patients who opted for the specialized PsoPlus consultation were younger and had longer disease duration. Decision parameters such as disease severity and quality of life were reported more often in the PsoPlus group. In the latter, a higher rate of patients were started on systemic therapy compared to the regular consultation group, and reporting on adverse events was done more frequently.

Conclusion: The implementation of a specialized consultation with comprehensive guidance facilitates documentation on disease-relevant parameters such as disease severity and quality of life. This format can be seen as a guidance for capturing data in a structured manner, with evidence showing that it significantly impacts treatment decision, treating not only psoriasis but the patient as a whole.

PsoPlus, a specialized consultation
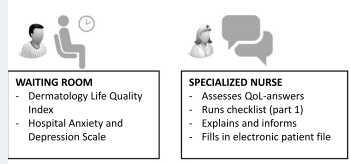

General consultation
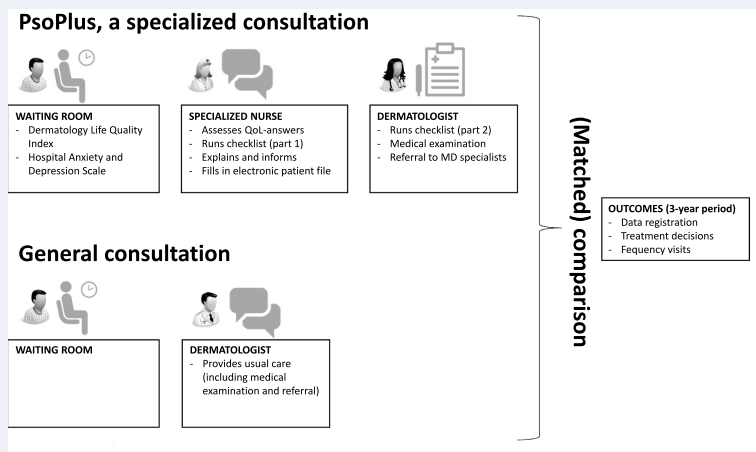

PsoPlus, a specialized consultation
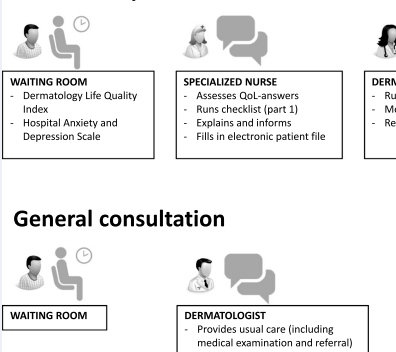

General consultation
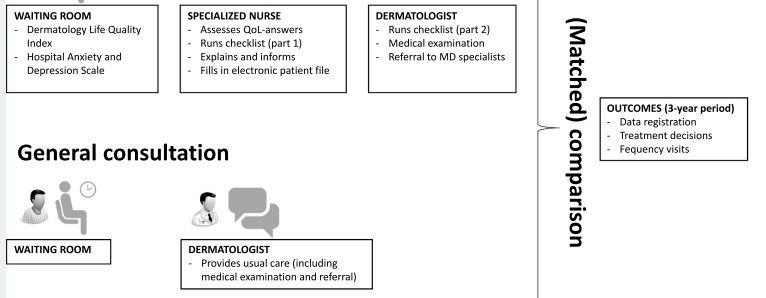

KEYWORDS

Structured consultation; specialized consultation; psoriasis; psoriasis management; value-based healthcare 


\section{Introduction}

Psoriasis is mainly known as a chronic skin condition characterized by elevated plaques of erythematous and scaly skin that may itch or burn. Psoriasis affects $2-4 \%$ of the world population [1] and has a negative impact on Quality of Life (QoL) and work productivity [2]. Although several compounds are available to treat psoriasis, there is no single drug which is effective in all cases. According to DISCOVER, a cross-sectional observational study in Belgium, nearly $40 \%$ of patients with moderate-to-severe psoriasis were undertreated despite the severity of their disease. This is caused by complextreatment decisions, leading to a substantial degree of undertreated and dissatisfied patients [3]. The rate of dissatisfaction with treatment is high among psoriasis patients, with higher dissatisfaction rates for exclusively topical treatments and lower dissatisfaction rates for treatment with biologics [4]. In addition, psoriasis is a multi-systemic disorder requiring insights that go beyond the skin as it is associated with both physical and mental comorbidities such as arthritis, metabolic syndrome and depression [5-10]. Therefore, an extensive and systematic patient anamnesis is required to have a good overview of all items for an optimal personalized approach.

To promote comprehensive management of psoriasis, some practice guidelines have been proposed. In 2013, a Spanish Working Group on Comorbidity in Psoriasis published guidelines for monitoring comorbidities in psoriasis [11]. One year later, Mrowietz and colleagues have proposed a shift towards 'management'-based psoriasis treatment, approaching skin inflammation and comorbidities (cardiovascular diseases and psychiatric disorders) together with negative consequences on QoL [5]. Finally, in 2016, our group (from Belgium) published a proposal for a multileveled consultation format for psoriasis [12]. In this work, an extensive consultation outline was generated, called PsoPlus, which addresses aspects beyond comorbidities and QoL in psoriasis that, in the end, affect management decisions. The consultation is executed by two specialists: a dedicated nurse and a dermatologist, each tackling a specific part of the psoriasis approach.

During the PsoPlus consultation, basic assessments such as disease severity and quality of life, are scored by the Psoriasis Area Severity Index (PASI) and the Dermatology Life Quality Index (DLQI) scales, respectively. Besides detailed demographic information, the patient is questioned about personal and family medical history, drug history, and current complaints. For example, one inquires into family planning, UV exposure, metabolic disorders, environmental influences, and previous and present psoriasis medication, which are all relevant in the light of tailored psoriasis management.
PsoPlus includes a checklist for clinical examination including aspects related to metabolic syndrome, e.g. body mass index and blood pressure, and further clinical investigations if required. This template can be integrated in an electronic patient file, which facilitates the collection of real-life data based on specific prefixed indices. The extent of the checklist also reminds the healthcare professional to inform the patient about educational aspects of psoriasis: it draws attention to seemingly unrelated aspects, such as UV exposure, vaccinations and mental health, as they are queried during the consultation. Importantly, the format urges a close follow-up and timely referrals to other specialists (e.g. rheumatology). In short, PsoPlus has the goal to increase the quality of the whole care cycle for psoriasis patients, hence improving treatment outcomes.

Belgium has no echeloning system, which means that any patient can attend a tertiary level clinic for diagnosis and treatment, regardless of disease severity, phenotype or drug history. In our department, two consultation formats coexist: at any time, psoriasis patients can choose if they visit either a PsoPlus consultation or a general consultation (not specifically for psoriasis, hereafter termed General consultation). The General consultation is the regular consultation format applied for all dermatological consultations in our department. In the General consultation, the dermatologist classically questions and fills out (electronically) four main information domains: medical history and current complaint, clinical examination, diagnosis and treatment. In the PsoPlus format, two healthcare professionals fill out a comprehensive psoriasis specific checklist, as described previously [12]. The PsoPlus consultation consequently takes forty minutes, twice as long as the General consultation.

PsoPlus consultation was implemented in the Dermatology Department of Ghent University Hospitalin December 2012. Although PsoPlus consultation guidance intuitively seems to be useful, its real impact and possible added value in the approach of psoriasis has not yet been evaluated yet. The first aim of this study was to describe the patient population who chooses for a specialized consultation. Secondly, an analysis was performed on the impact of the PsoPlus format in management decisions and outcome for the psoriasis patients.

\section{Methodology}

This paper presents the results of a 3-year implementation of PsoPlus. For the analysis, we focused on the impact of therapeutic approach of psoriasis and compared data from the PsoPlus consultation with data from General consultation for psoriasis at our premises. 
a

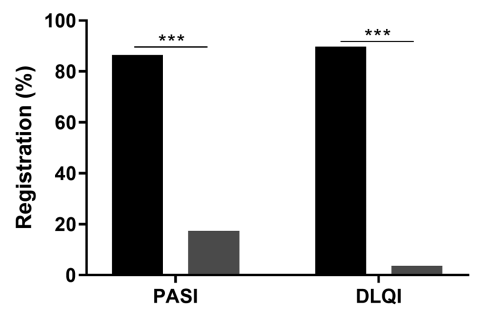

b
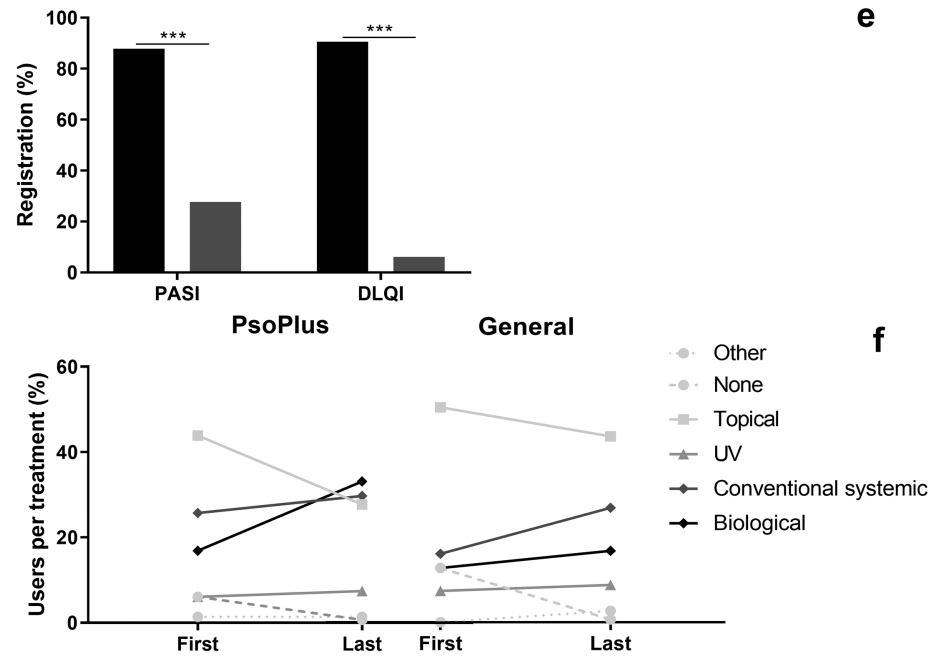

d

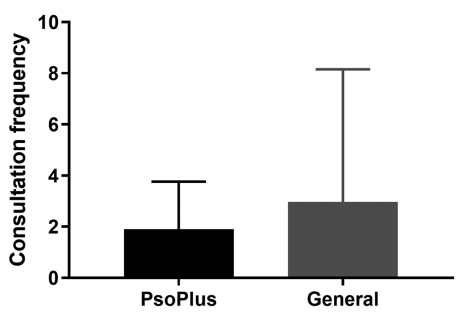

e
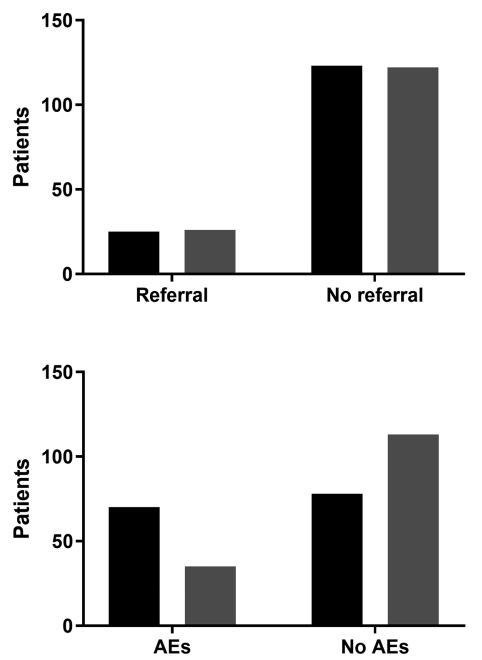

Figure 1. Comparison of PsoPlus and the general consultation. Patient files were screened for data on PASI and DLQI scores, treatment type, referrals, and number of consultations and adverse events. (a) and (b) Graphic visualization of registration rate of PASI, DLQI in both consultation formats in unmatched and matched patients, respectively. (c) Usage of medication type at the first and last consultation of the study period in PsoPlus (left) and General consultations (right). (d) Mean frequency of visits of matched patients (bars represent mean \pm SD). (e-f) Number of matched patients requiring referral to other specialists for comorbidities and number of patients reporting adverse events.Colours in bar graphs: black, PsoPlus consultation; grey, General consultation.

Table 1. Clinical characteristics of included patients.

\begin{tabular}{lcccc}
\hline & & PsoPlus & General & \\
& & 156 & 298 & p value \\
\hline Sex (male; \%) & 57.7 & 53.4 & 0.42 \\
Age (years. SD. range) & $45.1 \pm 15.2$ & $50.1 \pm 16.6$ & 0.002 \\
& & $(18-88)$ & $(19-89)$ & \\
Age group & $<18$ & 0.6 & 0 & $<0.001$ \\
(years; \%) & $18-35$ & 28.2 & 25.5 & \\
& $36-55$ & 48.1 & 33.6 & \\
Disease duration (years) & $14.6 \pm 11$ & $12 \pm 15.3$ & $<0.001$ \\
Phenotype (\%) & Vulgaris & $(0-51)$ & $(0-64)^{*}$ & \\
& CPPP & 3.9 & 65.5 & $<0.001$ \\
& Guttate & 3.9 & 13.6 & \\
& Inversa & 2 & 10.5 & \\
& Nail & 0.7 & 3.5 & \\
& Capitis & 0 & 4.9 & \\
& Capitis/ & 0 & 1.4 & \\
& nail & & 0.7 & \\
\hline
\end{tabular}

${ }^{*}$ Disease duration was noted in 210 of the General consultation patient files SD: standard deviation; CPPP: chronic palmoplantarpustulosis.

Furthermore, patient profiles were also taken into account when comparing both consultation formats.

We searched our database for patients with a diagnosis of psoriasis who came for consultation in the period of January 2013 until December 2015. We excluded patients who came for only one consultation in this period, patients who came more often to our department due to non-psoriasis skin problems (e.g. follow-up of non-melanoma skin cancer), patients who were participating in a clinical trial, patients who did not allow being included in the study and patients who came interchangeably for PsoPlus and General consultation. The included patients were subsequently divided into two groups: PsoPlus and General consultation group. This study was carried out in accordance with the principles expressed in the Declaration of Helsinki and written consent was obtained from all patients to analyse their data, and data was anonymized for analysis and publication. The project was approved by the Ghent University Hospital Ethics Committee (B6702017314642; $28^{\text {th }}$ of February 2017).

Data related to demography of patients (sex, age, disease duration, psoriasis phenotype), clinical evaluation (PASI, DLQI) and treatment choices were collected. If DLQI was missing in the General consultation, terms related to QoL, such as 'depression', 'anxiety', 'fatigue', 'embarrassment', or 'better QoL since the beginning of treatment' were sought for.Regarding treatment, we registered psoriasis therapy used throughout the study period, referral to other healthcare professionals, and frequency of visits. As we analyzed a period of followup (2013-2015), data from the first consultation refers to the first consultation from January 2013, which was not necessarily the first consultation at the department. Missing data is reported wherever applicable and omitted for final analysis. 
We performed statistical analysis comparing the two groups using SPSS Statistics 23. We used a ChiSquare test for analysis of the non-continuous variables, and a Mann-Whitney $U$ test for continuous variables. Selection of matched cases was done by propensity score matching in $\mathrm{R}$ (version 3.4.1) using the nearest neighbor matching method with logistic regression distance measure in the matchit function of the R-Package 'Matchit' (version 3.0.1). The results were considered statistically significant for $\mathrm{p}<0.05$.

\section{Results}

After searching our database, we found that from 998 psoriasis patients who came for consultation at our department between January 2013 and December 2015, 454 met the inclusion criteria: 156 from the PsoPlus group and 298 from the General consultation group.

\section{Population}

The two groups did not differ significantly in sex $(p=0.42$ ). The PsoPlus group was significantly younger, although this difference was small with an average age of 45.1 years in comparison to the General group (mean 50.1 years; Table 1).Additionally, disease duration was also significantly longer among PsoPluspatients (Table 1), though the difference was very limited(2.6 years) and having very limited to no impact on clinical decisions. Interestingly, phenotypes differed significantly between both consultation formats: PsoPlus patients were mostly patients with psoriasis vulgaris (almost 90\%) whereas in the General consultation group more than $30 \%$ of patients had other subtypes of psoriasis such as chronic palmoplantarpustular (CPPP), guttate, inversa, nail or capitis, as listed in Table 1.

\section{Clinical evaluation}

PASI and DLQI were registered significantly more in PsoPlus: $86.5 \%$ and $89.7 \%$ respectively in comparison to General consultation (17.4\% and $3.7 \%$ respectively; Figure 1(a); $\mathrm{p}<0.001)$.QoL-related terms in the General consultation were found in only $21.1 \%$ of the files.

\section{Therapy}

To compare treatment decisions in similar population groups, we matched population for sex, age, disease duration and phenotype. After matching, 296 patients were included, 148 patients from each group (Table 2). A similar trend was observed regarding the registration of PASI and DLQI amongst both groups (Figure 1(b); $p<0.001)$.
Table 2. Clinical characteristics after matching.

\begin{tabular}{|c|c|c|c|c|}
\hline & & $\begin{array}{c}\text { PsoPlus } \\
148\end{array}$ & $\begin{array}{c}\text { General } \\
148\end{array}$ & $\mathrm{p}$ value \\
\hline \multicolumn{2}{|l|}{ Sex (male; \%) } & 57.4 & 57.4 & 1.0 \\
\hline \multicolumn{2}{|c|}{ Age (years. SD. range) } & $\begin{array}{c}45.1 \pm 15.2 \\
(18-88)\end{array}$ & $\begin{array}{c}51.4 \pm 17.1 \\
(19-86)\end{array}$ & 0.001 \\
\hline Age group & $<18$ & 0.7 & 0.3 & 0.001 \\
\hline \multirow[t]{3}{*}{ (years; \%) } & $18-35$ & 27.7 & 25.0 & \\
\hline & $36-55$ & 48.6 & 39.9 & \\
\hline & $>55$ & 23.0 & 34.8 & \\
\hline \multicolumn{2}{|c|}{ Disease duration (years) } & $\begin{array}{c}14.7 \pm 11 \\
(0-51)\end{array}$ & $\begin{array}{c}14.6 \pm 16.0 \\
(0-64)\end{array}$ & 0.035 \\
\hline \multirow[t]{7}{*}{ Phenotype (\%) } & Vulgaris & 91.2 & 89.2 & 0.61 \\
\hline & CPPP & 3.4 & 4.7 & \\
\hline & Guttate & 3.4 & 4.1 & \\
\hline & Inversa & 1.4 & 0.7 & \\
\hline & Nail & 0.7 & 1.4 & \\
\hline & Capitis & 0 & 0 & \\
\hline & $\begin{array}{l}\text { Capitis/ } \\
\text { nail }\end{array}$ & 0 & 0 & \\
\hline
\end{tabular}

SD: standard deviation; CPPP: chronic palmoplantarpustulosis.

Next, we noted the treatment types at the initiation and end of the follow-up and found that treatment decisions differed significantly $(p<0.001)$ between the two groups, depicted in Figure 1(c). Systemic therapies, including conventional and biologicals, were more frequently prescribed in PsoPlus towards the last registered consultation: $62.8 \%$ of patients in PsoPluscompared to $43.9 \%$ in General consultation. Exclusive use of topical treatment remained high in the General consultation (from $50.7 \%$ to $43.9 \%$ ) whereas this was greatly reduced in the PsoPlus cohort (from $43.9 \%$ to $27.7 \%$ ). The prescription of biologicals showed a steep increase in the PsoPlus group (from $16.9 \%$ to $33.1 \%)$. This was not observed in patients from the General consultation, where only a small increase was observed (4.1\%). In contrast, conventional drugs such as methotrexate and cyclosporine were more frequently prescribed in the General group.

Finally, over a period of 3 months PsoPlus patients had less frequent visits, yet this was not significant amongst the matched cases (1.4 versus 2.9 consultations in General, $p=0.16$; Figure 1(d)). In the unmatched population this was significantly different ( $p=0.0188$; data not shown). The percentage of patients who were referred to other healthcare professionals was similar in both groups (16.9\% PsoPlus and $17.6 \%$ General consultation, $\mathrm{p}=0.87$ ) (Figure 1(e)). Remarkably, side effects were more prevalent in thePsoPlus group (47.3\% versus $23.6 \%$ in the General, $p<0.001$; Figure 1(f)). PsoPlus patients were thus more likely to experience adverse events, with an odds ratio of 2.897 (95\% confidence interval: 1.758-4.804, $\mathrm{p}<0.0001)$.

\section{Discussion}

PsoPlus is a guided consultation outline with a comprehensive checklist to be completed by both a specialized nurse and a dermatologist. The checklist 
comprises important aspects to be considered in the evaluation of psoriasis patients, aiming to promote a more personalized approach. In addition,we show that the use of a structured and pre-defined electronic patient file arranged as a checklist can be useful for treatment decisions. Based on the low number of missing data, we show that rigorous documentation of psoriasis-related parameters is feasible in a center of excellence. Due to the inherent infrastructure of the General consultation, it was not possible to compare clinical outcome measures such as PASI scores between both consultation formats. We were however successful in providing evidence that the General consultation lacks the rigorous registration of parameters, such as PASI and DLQI, which are crucial for treatment decisions. Especially for biologicals: the Belgian reimbursement system requires a minimal PASI score of 10, which as a consequence of not registering PASI scores, leads to a subjective assessment of disease severity and treatment progress by the physician [13]. DLQI scores can be used as additional arguments to indicate the severity of a patient's case from a QoL-point of view. Hence, we interpret that the steep increase in PsoPlus-patients on biologicals is presumably supported by the consistent registration of PASI and DLQI scores, facilitating treatment decision to initiate the approval process to get reimbursement for biologicals. Only 7 patients from the General consultation were started on biologicals in the study period, whereas only 4 out of these 7 patient files (57.1\%) includeda PASI score. Furthermore, conventional drugs were still considered in the PsoPlus cohort during treatment management. However, after affirming whether previous use of conventional drugs was met with either adverse events or insufficient clinical response; patients were found eligible for biologicals according to the Belgian regulation. Therefore, it is plausible that due to the consistent registration of potential contra-indications, PsoPlus patients were found more often eligible for treatment with biologicals, therefore favouring this type of treatment in this group.

The increased prevalence of adverse events in PsoPlus can be attributed to different reasons. First, both conventional and biological drugs were more prescribed in the PsoPlus cohort, partially explaining the higher likelihood to adverse events. Next, PsoPlus patients are actively asked about safety and tolerability of their current treatments in accordance with the specific tabs in the PsoPlus patient files. Lastly, in contrast to the General group, patients are first seen by a nurse during PsoPlus. There is a possibility that the communication with a nurse is easier and thus reveals more information on uncomfortable experiences with treatments than with a dermatologist.

Importantly, PsoPlus was visited less frequently, which is an asset for chronic diseases such as psoriasis.
This is especially valuable for patients who have daytime jobs, as consultations are often planned during the working hours. As a consequence, patients with chronic diseases are often forced to sacrifice holidays for doctor visits. An additional reason for less frequent consultations may be that withinPsoPlus the possibility of contact via email in between consultations is provided, enabling the patient to inquire more information related to treatment or share certain concerns with a healthcare professional.This remains to be confirmed in a prospective study.

The data presented here are based on matched populations, correcting for bias such as different phenotypes - which may impact treatment decision. However, we acknowledge that this study has limitations, particularly the lack of prospective data. PASI scores were not consistently registered in the General Consultation, making a direct comparison for disease severity not possible with the PsoPlus cohort. This could explain why biologicals were prescribed more often in the PsoPlus group. Additionally, this study lacks a cross-over design in which we inquire into the patient's experience and satisfaction during the General and PsoPlus consultations; an aspect that should be taken into account. Finally, patients could freely choose for PsoPlus or the General consultation, and hence were not randomized. It has been postulated that PsoPlus may attract more moderate-severe cases. However, we argue that mild cases presented to PsoPlus as well as $45.9 \%$ of PsoPlus-patients had an initial PASI score of less than 10 . We believe this is indicative that patients with mild psoriasis may experience a great impact on their quality of life $[14,15]$ and therefore opt for specialized psoriasis care

Long-term and prospective data are required to evaluate in-depth the advantages of a structured consultation format such as PsoPlus. In addition, other parameters, such as absenteeism and indirect costs for the patient, should be taken into consideration if cost-effectiveness is to be evaluated. Such concept can be found in value-based healthcare, where patients enter a care cycle that measures the overall impact of the given care, beyond PASI and DLQI. Additional impact on absenteeism or comorbidities provide more value to the patient and the overall healthcare system both on the short and long term. In this context, a comprehensive and specialized consultation format such as PsoPlus poses an interesting tool to investigate value-based healthcare in psoriasis.

\section{Conclusion}

In conclusion, the implementation of a structured and specialized consultation format encompassing all aspects of psoriasis is feasible in clinical practice and facilitates documentation on disease-relevant parameters such as disease severity and quality of life. 
Moreover, this global clinical patient evaluation can significantly impact treatment decision, in order to adjust the psoriasis management, thereby treating not only psoriasis but the patient as a whole.

\section{Disclosure statement}

The authors report no conflicts of interest.

\section{Funding}

AKAMwas funded by a PhD Fellowship of the agency of Innovation by Science and Technology (IWT)-TGO under Grant [120829], Belgium.

\section{References}

[1] Parisi R, Symmons DPM, Griffiths CEM, et al. Global epidemiology of psoriasis: a systematic review of incidence and prevalence. J Invest Dermatol. [Internet]. 2013 [cited 2014 Jul 11];133(2):377-385. Available from: http://www.ncbi.nlm.nih.gov/pubmed/ 23014338.

[2] Korman NJ, Zhao Y, Pike J, et al. Relationship between psoriasis severity, clinical symptoms, quality of life and work productivity among patients in the USA. Clin Exp Dermatol. 2016;41(5):514-521.

[3] Lambert J, Ghislain PD, Lambert J, et al. Treatment patterns in moderate to severe plaque psoriasis results from a Belgian cross sectional study DISCOVER. J Dermatolog Treat. 2016.

[4] Vaidya TS, Alikhan A. Treatment satisfaction among patients with psoriasis at a large academic center: an observational study. J Dermatolog Treat. 2017;28 (4):288-289.

[5] Mrowietz U, Steinz K, Gerdes S. Psoriasis: to treat or to manage? Exp Dermatol. [Internet]. 2014 [cited 2014 Sep 25];1-5. Available from: http://www.ncbi.nlm.nih. gov/pubmed/24815425.

[6] Boehncke S, Thaci D, Beschmann H, et al. Psoriasis patients show signs of insulin resistance. $\mathrm{Br}$ J Dermatol. [Internet]. 2007 [cited 2015 May 19];157 (6):1249-1251. Available from: http://www.ncbi.nlm. nih.gov/pubmed/17916217
[7] Suárez-Fariñas M, Li K, Fuentes-Duculan J, et al. Expanding the psoriasis disease profile: interrogation of the skin and serum of patients with moderate-tosevere psoriasis. [Internet]. J Invest Dermatol. 2012 [cited 2014 May 13];132(11):2552-2564. Available from: http://www.pubmedcentral.nih.gov/articleren der.fcgi?artid=3472561\&tool=pmcentrez\&render type $=$ abstract

[8] Ni C, Chiu MW. Psoriasis and comorbidities: links and risks. Clin Cosmet Investig Dermatol. [Internet]. 2014 [cited 2014 Aug 10];7:119-132. Available from: http:// www.pubmedcentral.nih.gov/articlerender.fcgi?artid= 4000177\&tool=pmcentrez\&rendertype=abstract.

[9] Radtke MA, Mrowietz U, Feuerhahn J, et al. Early detection of comorbidity in psoriasis: recommendations of the national conference on healthcare in psoriasis. J Dtsch Dermatol Ges. [Internet]. 2015;13:674-689.

[10] Bostoen J, Van Praet L, Brochez L, et al. A cross-sectional study on the prevalence of metabolic syndrome in psoriasis compared to psoriatic arthritis. J Eur Acad Dermatol Venereol. [Internet]. 2014;28 (4):507-511. Available from: http://www.ncbi.nlm.nih. gov/pubmed/24772493

[11] Daudén E, Castañeda S, Suárez C, et al. Clinical practice guideline for an integrated approach to comorbidity in patients with psoriasis. J Eur Acad Dermatol Venereol. 2013;27(11):1387-1404.

[12] DeCoster E, Alves de Medeiros A, Bostoen J, et al. A multileveled approach in psoriasis assessment and follow-up: A proposal for a tailored guide for the dermatological practice. J Dermatolog Treat. [Internet]. 2016;27(4):298-310. Available from: http://www.tand fonline.com/doi/full/10.3109/09546634.2015.1117566

[13] Feldman SR, Krueger GG. Psoriasis assessment tools in clinical trials. Ann Rheum Dis. 2005;64(Suppl 2):ii65-8. discussion ii69-73.

[14] Mermin D, Boursault L, Milpied B, et al. DLQI as a major criterion for introduction of systemic agents in patients with mild psoriasis. J Eur Acad DermatolVenereol. 2016;30:1961-1964.

[15] Misery L, Thomas L, Jullien D, et al. Comparative study of stress and quality of life in outpatients consulting for different dermatoses in 5 academic departments of dermatology. Eur J Dermatol. [Internet]. [cited 2018 May 8];18:412-415. Available from: http://www.ncbi. nlm.nih.gov/pubmed/18573714 